\title{
Os desafios enfrentados pelos profissionais da enfermagem frente à COVID-19
}

\author{
The challenges faced by nursing professionals facing COVID-19 \\ Los desafíos que enfrentaron los profesionales de enfermería durante el COVID-19
}

Recebido: 06/11/2021 | Revisado: 12/11/2021 | Aceito: 14/11/2021 | Publicado: 24/11/2021

Jackeline Sousa Ribeiro

ORCID: https://orcid.org/0000-0001-9426-6923 Faculdade de ensino Superior da Amazônia Reunida, Brasil E-mail: ribeirojacke2611@gmail.com

Millena Rhandara Silva Donizete ORCID: https://orcid.org/0000-0003-3578-0871 Faculdade de ensino Superior da Amazônia Reunida, Brasil E-mail: millenaenf2017@gmail.com

Amanda Augusta de Paula

ORCID:https://orcid.org/0000-0002-6215-182X

Faculdade de ensino Superior da Amazônia Reunida, Brasil

E-mail: amandaaugusta10@ hotmail.com

Jaianny Macedo Chaves

ORCID: https://orcid.org/0000-0001-9638-7614 Faculdade de ensino Superior da Amazônia Reunida, Brasil E-mail: jaiannymacedo2217@gmail.com

Aline de Oliveira Vieira

ORCID: https://orcid.org/0000-0002-5620-6513 Faculdade de ensino Superior da Amazônia Reunida, Brasil E-mail: alineoly11@hotmail.com

Laiza Andressa Silva Pereira

ORCID: https://orcid.org/0000-0003-4082-2335 Faculdade de ensino Superior da Amazônia Reunida, Brasil E-mail: laiza.pereira@ fesar.edu.br

\begin{abstract}
Resumo
O objetivo desse trabalho foi identificar os desafios enfrentados pelos profissionais da enfermagem durante a pandemia da COVID-19, realizado através de um estudo de revisão integrativa da literatura com abordagem qualitativa, realizouse a revisão da literatura de artigos publicados nas bases de dados Scientific Electronic Library Online (SCIELO), Biblioteca virtual em saúde (BVS), National Library of medicine National institutis of Health (PUBMED), Literatura Latino-americana do Caribe em Ciências da Saúde (LILACS), o processo de seleção e triagem dos estudos foi efetuado por meio da leitura minuciosa de títulos e resumos, seguindo os critérios de seleção previamente estabelecidos que foram: artigos de acesso online aberto, no idioma de linguagem portuguesa e que contemplaram os profissionais da saúde que atuaram na linha de frente a COVID-19, com ênfase na equipe de enfermagem. Para a pesquisa inicial foi utilizado 42 artigos, após a leitura na integra, foram excluídos 33 artigo, restando 9 artigos de acordo com a relevância para a revisão integrativa. A partir dos artigos analisados e selecionados, os autores elaboraram um quadro que contemplam informações dos artigos estudados, como: título, autores, ano de publicação, população e os resultados apresentados. Conclui-se que a equipe de multiprofissionais que compõem o sistema de saúde, sobretudo a enfermagem, vivenciaram um momento desafiador com o surgimento da COVID-19, tais desafios ocasionou vários problemas a esses trabalhadores, tanto a nível físico como psicológico.
\end{abstract}

Palavras-chave: Profissionais de enfermagem; Desafios; COVID-19.

\begin{abstract}
The objective of this work was identifying the challenges faced by nursing professionals during the COVID-19 pandemic. This is an integrative review study with a qualitative approach, a literature review of articles published in the Scientific Electronic Library Online (SCIELO), Virtual Health Library (BVS), National Library of Medicine, National Institutes of Health (PUBMED) databases ), Caribbean Latin American Literature on Health Sciences (LILACS), the selection and screening process of the studies was carried out through the thorough reading of titles and abstracts, following the previously established selection criteria, which were: articles for online access open, in the Portuguese language and which included the health professionals who worked on the frontline of COVID-19, with an emphasis on the nursing team. For the initial research 42 articles were used, after reading in full, 33 articles were excluded, leaving 9 articles according to their relevance for the integrative review. From the analyzed and selected articles, the authors elaborated a table that includes information from the studied articles, such as: title, authors, year of publication, population and the results presented. The multidisciplinary team that makes up the health system, especially nursing, experienced a challenging moment with the emergence of COVID-19, such challenges caused several problems for
\end{abstract}


these workers, among them the most mentioned psychological disorders.

Keywords: Nursing professionals; Challenges; COVID-19.

\section{Resumen}

El objetivo de este trabajo fue identificar los desafíos que enfrentaron los profesionales de enfermería durante la pandemia COVID-19, realizado a través de un estudio de revisión integradora con un enfoque cualitativo, se realizó una revisión de la literatura de los artículos publicados en las bases de datos Scientific Electronic Library Online (SCIELO), Biblioteca Virtual en Salud (BVS), Biblioteca Nacional de Medicina, Institutos Nacionales de Salud (PUBMED), Literatura del Caribe Latinoamericano en Ciencias de la Salud (LILACS), el proceso de selección y cribado de los estudios se realizó mediante la lectura minuciosa de títulos y resúmenes, siguiendo los criterios de selección previamente establecidos, que fueron: artículos de acceso abierto en línea, en idioma portugués y que incluyeron a los profesionales de la salud que trabajaron en la primera línea del COVID-19, con énfasis en el equipo de Enfermería. Para la investigación inicial se utilizaron 42 artículos, luego de la lectura completa se excluyeron 33 artículos, quedando 9 artículos según su relevancia para la revisión integradora. A partir de los artículos analizados y seleccionados, los autores elaboraron una tabla que incluye información de los artículos estudiados, tales como: título, autores, año de publicación, población y los resultados presentados. Se concluye que el equipo multidisciplinario que conforma el sistema de salud, en especial la enfermería, vivió un momento desafiante con el surgimiento del COVID-19, tales desafíos ocasionaron varios problemas a estos trabajadores, entre ellos los trastornos psicológicos más mencionados.

Palabras clave: Profesionales de enfermería; Desafíos; COVID-19.

\section{Introdução}

O mundo tem passado por um momento de desafio no contexto da saúde pública após o surgimento da COVID-19, que é causada pelo vírus da síndrome respiratória aguda (SARS-CoV-2), que se configura como uma nova enfermidade em todo o mundo. A COVID-19 trata-se de uma doença com rápido perfil de transmissibilidade entre indivíduos e seu maior potencial para a infecção é determinado pelas gotículas espalhadas pelo ar, através de pessoas contaminadas, podendo desenvolver os sintomas característicos da doença ou não, alguns indivíduos são assintomáticos ao vírus (Ferreira et al., 2020; Pereira et al., 2020; Santos et al., 2020).

Em dezembro de 2019 a doença foi relatada pela primeira vez em Wuhan, na China e em fevereiro de 2020 foi notificado o primeiro caso no Brasil. O respectivo vírus chegou ao Brasil, acarretando sobrecarga aos serviços de saúde e afetando diretamente os trabalhadores desse setor, os quais têm lutado incansavelmente nos cuidados aos pacientes e na contenção do vírus (Humerez et al., 2020).

Dentre os profissionais da saúde que atuam na linha de frente, os enfermeiros, técnicos de enfermagem e auxiliares de enfermagem, veem se dividindo em turnos exaustivos de trabalho e enfrentando sérias implicações para controlar a demanda de atendimentos aos pacientes. A enfermagem já exerce naturalmente o papel da educação continuada dentro da unidade hospitalar, no entanto, vem atuando na prevenção e no tratamento de pacientes contaminados pelo vírus. Assim, o momento faz com que o mundo reconheça a importância do papel desenvolvido pelos profissionais de saúde, do enfermeiro e da enfermagem (Barbosa et al., 2020; Toescher et al., 2020).

As situações enfrentadas pela equipe de enfermagem no decorrer da pandemia, ocasionou medo e insegurança, gerando fatores de desequilíbrio a saúde física e mental dos trabalhadores. A exaustão física e mental desses trabalhadores e a dificuldade em conciliar com sua vida fora do ambiente hospitalar, potencializam o aumentando do risco para o adoecimento (Queiroz et al., 2021; Teixeira et al., 2020; Santana et al., 2020).

Diante desse cenário, a presente pesquisa tem por finalidade identificar os desafios enfrentados pelos profissionais da enfermagem durante a pandemia da COVID-19, correlacionando os fatores associados à sobrecarga de trabalho e os fatores que levaram a contaminação dos profissionais de enfermagem no contexto atual.

\section{Metodologia}

Trata-se de uma pesquisa de revisão integrativa da literatura com abordagem qualitativa, que permite a formulação de 
conclusões gerais a respeito de determinada área do conhecimento, mediante síntese de estudos publicados anteriormente (Botelho et al., 2011; Souza et al., 2010)

Para a construção do estudo, os autores seguiram etapas distintas, sendo elas divididas em seis passos: identificação do tema, pergunta norteadora do estudo, estabelecimento de critérios inclusivos e exclusivos da pesquisa, identificação dos estudos pré-selecionados e selecionados, categorização dos estudos selecionados, análise e interpretação dos resultados, e por fim, apresentação da revisão e síntese do conhecimento.

A coleta de dados ocorreu entre agosto e setembro de 2021, através dos bancos de dados: Scientific Electronic Library Online (SCIELO), Biblioteca virtual em saúde (BVS), National Library of medicine National institutis of Health (PUBMED), Literatura Latino-americana do Caribe em Ciências da Saúde (LILACS). Para a busca foram utilizados descritores como "Profissionais de Enfermagem; Desafios; e COVID-19", os artigos foram selecionados com datas de publicação entre janeiro de 2020 a setembro de 2021.

O processo de seleção e triagem dos estudos foi efetuado por meio da leitura minuciosa dos títulos e resumos, seguindo os critérios de seleção previamente estabelecidos que foram: artigos de acesso online aberto, no idioma de linguagem portuguesa e que contemplavam a temática dos profissionais da saúde que atuaram na linha de frente a COVID-19, com ênfase na equipe de enfermagem. E os artigos excluídos foram os que não abordaram profissionais de enfermagem atuantes na linha de frente da COVID-19, artigos duplicados, incompletos, e de outra natureza.

Os artigos previamente selecionados foram analisados através de uma ficha analítica adaptada, conforme descrito por Ursi e Galvão (2006), que abordou as seguintes informações: título periódico, título do artigo, autores, ano de publicação, objetivos do estudo e principais resultados.

\section{Resultados}

A pesquisa inicial identificou 42 artigos selecionados, após a leitura dos títulos e resumos, foram excluídos quatro estudos que não se incluíam na temática abordada, totalizando 38 artigos para triagem inicial. Posteriormente, foi realizada leitura na integra dos estudos previamente selecionados, observando os critérios de inclusão e exclusão da pesquisa, nessa etapa foram excluídos 29 artigos, por apresentarem estudos incompletos; fuga da temática; estudos com duplicatas e estudos que não se caracterizavam como artigos científicos. Para esta pesquisa, foram selecionados 9 artigos de acordo com a relevância para a revisão integrativa, conforme o Fluxograma 1. A partir dos artigos analisados e selecionados, os autores elaboraram um quadro que contemplam informações dos artigos estudados como: título, autores, ano de publicação, população e os resultados apresentados. (Quadro 1). 
Research, Society and Development, v. 10, n. 15, e186101522918, 2021

(CC BY 4.0) | ISSN 2525-3409 | DOI: http://dx.doi.org/10.33448/rsd-v10i15.22918

Fluxograma 1. Etapas metodológicas utilizadas para a seleção dos artigos.
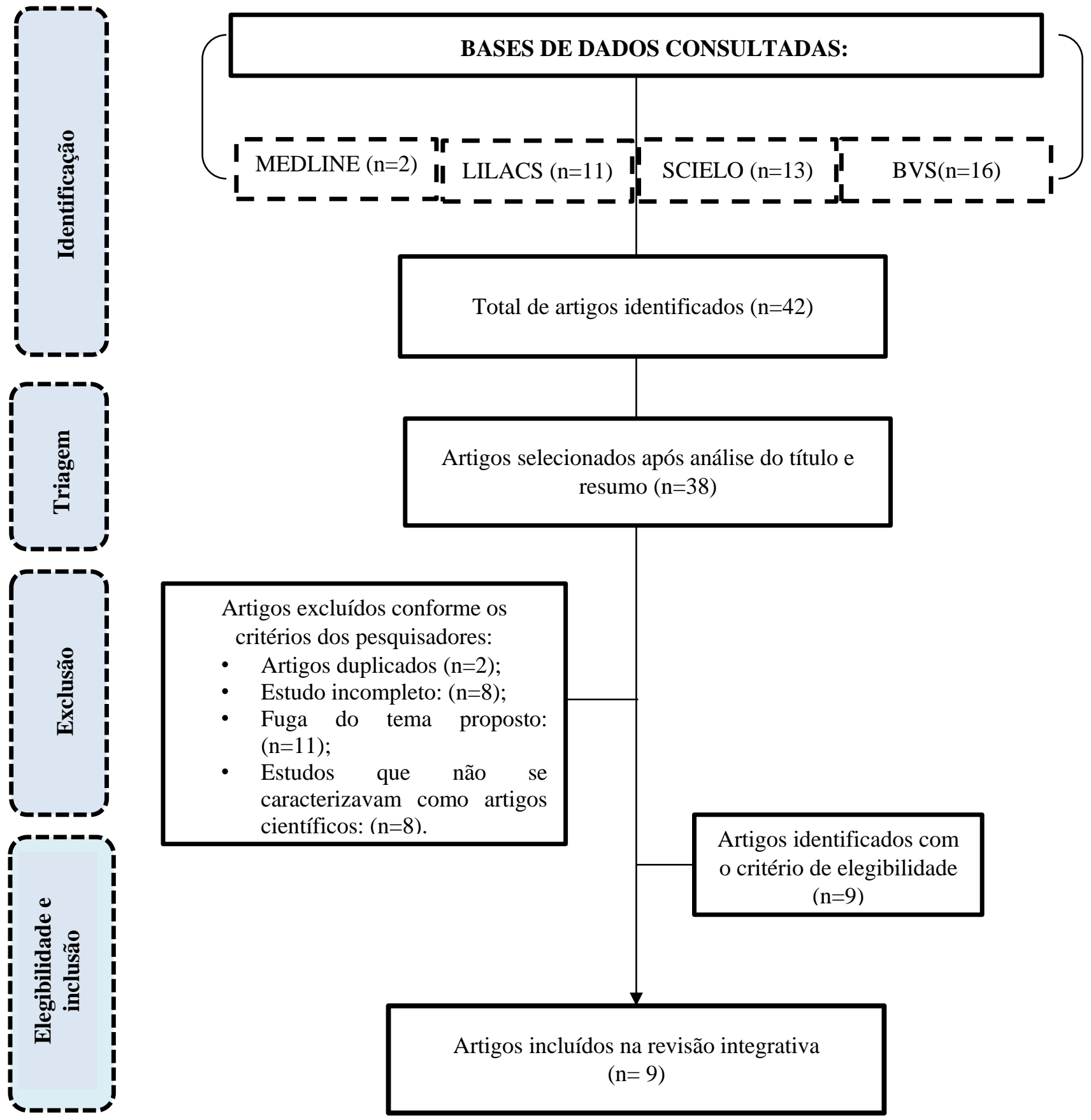

Total de artigos identificados $(n=42)$

Artigos selecionados após análise do título e resumo $(\mathrm{n}=38)$

Artigos excluídos conforme os critérios dos pesquisadores:

- $\quad$ Artigos duplicados $(\mathrm{n}=2)$;

- Estudo incompleto: $(\mathrm{n}=8)$;

- Fuga do tema proposto: $(n=11)$;

- Estudos que não se caracterizavam como artigos científicos: $(n=8)$

Artigos identificados com o critério de elegibilidade $(\mathrm{n}=9)$

Artigos incluídos na revisão integrativa $(n=9)$

Fonte: Autores. 
Quadro 1: Artigos selecionados pelos pesquisadores para a revisão integrativa.

\begin{tabular}{|c|c|}
\hline & $\begin{array}{c}\text { TÍTULO } \\
\text { AUTORES /ANO } \\
\text { TIPO DE ESTUDO } \\
\text { POPULAÇÃO DO ESTUDO } \\
\text { OBJETIVO DO ESTUDO } \\
\text { PRINCIPAIS RESULTADOS }\end{array}$ \\
\hline Miranda et al., (2020). & $\begin{array}{l}\text { Sofrimento psíquico entre os profissionais de enfermagem durante a pandemia da covid-19: Scoping } \\
\text { Review } \\
\text { Revisão sistemática } \\
\text { Médicos e enfermeiros }\end{array}$ \\
\hline Moreira \& Lucca, (2020). & $\begin{array}{l}\text { Apoio psicossocial e saúde mental dos profissionais de enfermagem no combate à covid-19. } \\
\text { Revisão narrativa } \\
\text { Profissionais de enfermagem }\end{array}$ \\
\hline Horta et al., (2021). & $\begin{array}{l}\text { O estresse e a saúde mental de profissionais da linha de frente da COVID-19 em hospital geral. } \\
\text { Recorte transversal de estudo prospectivo } \\
\text { Profissionais da saúde }\end{array}$ \\
\hline Góes et al., (2020). & $\begin{array}{l}\text { Desafios de profissionais de Enfermagem Pediátrica frente à pandemia da COVID-19. } \\
\text { Pesquisa descritiva e exploratória, de abordagem qualitativa. } \\
\text { Profissionais de enfermagem. } \\
\text { COVID-19. } \\
\text { Identificar os desafios que os profissionais da enfermagem pediátrica enfrentaram no combate da } \\
\text { O estudo mostra que os profissionais de enfermagem relataram falta de equipamentos de proteção } \\
\text { individual, falta de treinamentos e preocupação com os colegas de trabalho. Durante o combate a COVID- } \\
\text { 19, a falta de conhecimento e informações relacionadas à doença, ocasiona medo e insegurança. }\end{array}$ \\
\hline Ludwig et al., (2020). & $\begin{array}{l}\text { Pandemia da COVID-19: percepção dos profissionais de saúde sobre a assistência aludida em mídia } \\
\text { televisiva. } \\
\text { Estudo exploratório e descritivo, do tipo documental, qualitativo. } \\
\text { Profissionais de saúde. } \\
\text { Apresentar a percepção dos profissionais de saúde sobre a assistência prestada durante a pandemia da } \\
\text { COVID-19, relatado em mídia televisiva. } \\
\text { O artigo cientifico refere aos profissionais da saúde uma mobilidade subjetiva diante da sobrecarga que } \\
\text { vem enfrentando, que resulta em sentimentos de exaustão, medo, tristeza, insegurança e fadiga física e } \\
\text { emocional. }\end{array}$ \\
\hline
\end{tabular}




\begin{tabular}{|c|c|}
\hline Borges et al., (2021). & $\begin{array}{l}\text { Percepções e vivências de enfermeiros sobre o seu desempenho na pandemia da covid-19 } \\
\text { Estudo qualitativo, com abordagem fenomenológica. } \\
\text { Profissionais de enfermagem. } \\
\text { Descrever as vivências e percepções dos enfermeiros em relação ao seu desempenho durante a pandemia } \\
\text { da COVID-19. } \\
\text { Estudo traz os desafios e vivencias enfrentados pela equipe de enfermagem como estado emocional } \\
\text { negativo associados a ansiedade, angustia, medo a exposição ao vírus, preocupação e insegurança. } \\
\text { Ressaltaram as instalações precárias no ambiente de trabalho dificultando a realização do seu serviço. }\end{array}$ \\
\hline Alves \& Ferreira, 2020. & $\begin{array}{l}\text { COVID-19: Reflexão da atuação do enfermeiro no combate ao desconhecido. } \\
\text { Estudo de reflexão bibliográfica. } \\
\quad \text { Profissionais de enfermagem. } \\
\text { Refletir as consequências na atuação do enfermeiro diante do surgimento da covid-19. } \\
\text { O estudo descreve a dificuldade dos profissionais de enfermagem em lidar com o desconhecido, onde não } \\
\text { estavam preparados para a pandemia, os principais desafios e a busca por conhecimento e controlar a } \\
\text { contaminação em massa do vírus. }\end{array}$ \\
\hline Reis et al., (2020). & $\begin{array}{l}\text { Atuação da enfermagem no cenário da pandemia COVID-19. } \\
\text { Relato de experiência com abordagem qualitativa. } \\
\text { Profissionais de enfermagem. }\end{array}$ \\
\hline Souza et al., (2020). & $\begin{array}{l}\text { Trabalho de enfermagem na pandemia da covid-19 e repercussões para a saúde mental dos trabalhadores. } \\
\text { Estudo teórico-reflexivo. } \\
\text { Profissionais de enfermagem. }\end{array}$ \\
\hline
\end{tabular}

Fonte: Autores.

\section{Discussão}

Segundo Alves e Ferreira (2020), relataram que a pandemia tornou um cenário desafiador para os sistemas de saúde em todo mundo, bem como afetou diretamente os profissionais de saúde que compõem esse sistema, fazendo com que surgissem diversos novos desafios a serem enfrentados. Moreira e Lucca (2020), ressaltaram que os profissionais de saúde por estarem diretamente em contato com o paciente no ambiente hospitalar e por sua jornada de trabalho no enfrentamento ao desconhecido, estão mais suscetíveis a desenvolver doenças tanto a nível físico como psicológico. Com o aumento da demanda gerada pelos serviços de saúde ocasionados pela pandemia, à carga horária de trabalho desses profissionais se intensificou e desencadeou diversos problemas de saúde aos mesmos. Borges et al. (2020), descreveram um conjunto de fatores distintos que impactaram diretamente na saúde e bem estar dos profissionais atuantes na pandemia, com relação aos profissionais da enfermagem, público esse, que estão ligados ao cuidado e promoção a saúde do paciente, são mais susceptível a infecção pelo vírus.

Em seu estudo, Alves e Ferreira (2020), relataram que a sobrecarga de trabalho gerou grandes desafios para os enfermeiros, em decorrência ao aumento do número de pacientes internados e o afastamento de colegas de profissão contaminados pelo vírus, assim acarretando prejuízos à saúde desses profissionais. Borges et al. (2021), enfatizou que a pandemia acarretou aos enfermeiros desafios associados ao trabalho e a busca pelo conhecimento, contudo, a procura por novas informações foi identificada como um período desafiador para a equipe.

Reis et al. (2020), destacou o risco que os profissionais de saúde enfrentaram no trabalho devido à falta de capacitação da equipe, sendo um dos fatores de alto índice de contaminação entre eles. O aumento dos atendimentos na unidade hospitalar, tem 
ocasionado escassez dos Equipamentos de Proteção Individual (EPI's) relacionado à falta de insumos para a fabricação. A utilização incorreta dos equipamentos durante o manejo ao paciente e a desparamentação também é um forte fator de contaminação. As condições precárias de trabalho que são geradas por infraestrutura inadequada, ocasionou maior vulnerabilidade para esses trabalhadores. O estresse e a necessidade do uso contínuo de EPI's, somados com as angústias para tomar decisões difíceis durante o trabalho, tornaram-se fatores estressores no enfrentamento da pandemia (Ludwig et al., 2021).

A pandemia do novo coronavírus impactou diretamente a saúde mental desses trabalhadores, principalmente a equipe de enfermagem, onde passam horas de suas vidas dentro do ambiente hospitalar, gerando em si medo e insegurança. As longas horas de trabalhos que são submetidos ao uso de EPI's, são fatores estressantes para os profissionais. O medo e insegurança de levar o vírus para casa e contaminar familiares e amigos resultam em quadro de estresse, depressão e consequentemente síndrome de Burnout (Góes et al., 2020; Horta et al., 2021). Souza et al. (2020), identificaram que as condições de trabalho foi um fator determinante para o sofrimento psíquico dos trabalhadores, as situações inadequadas de trabalho geram insegurança, comprometendo o atendimento prestado ao paciente.

Miranda et al. (2020), aponta que para tentar amenizar o estresse e sofrimento psíquico gerado a equipe, as instituições de saúde precisam implementar ações que capacitem cada dia mais sua equipe de trabalhadores, bem como assistência e apoio psicossocial em curto espaço de tempo, gerando proteção e segurança aos prestadores de serviço. No entanto, tais estratégias necessitam ser definias, o que exige investimento financeiro continuo, acompanhamento da saúde mental desses profissionais, bem como, nas instituições públicas e privadas.

\section{Conclusão}

A enfermagem e a equipe de multiprofissionais que compõem os sistemas de saúde vivenciaram um momento desafiador após o surgimento da COVID-19, e enfrentaram diversas dificuldades como elencado pelos artigos selecionados nessa revisão integrativa. Onde pode-se concluir que após o surgimento da pandemia, a carga horária de trabalho desses profissionais se intensificou, levando-os a ficarem por mais tempo expostos a variados riscos devido as condições inapropriadas para o trabalho, como a escassez de equipamentos de proteção individual (EPI's) e infraestrutura inadequada. O que gerou sentimentos de medo e insegurança a esses profissionais, ocasionando problemas físicos e emocionais.

Portanto, para reduzir tais problemas, sobretudo mentais, medidas preventivas se fazem necessárias. O apoio psicossocial pode ser uma medida eficaz para que consigam lidar melhor com a excessiva carga de trabalho e prevenir o esgotamento físico destes profissionais. Além, de que tais medidas podem refletir diretamente na qualidade do serviço prestado.

Cabe ressaltar, que o desenvolvimento de protocolos institucionais é importante para realização de uma assistência mais segura e ampla aos pacientes, assim como, para os profissionais. Para tanto, observa-se a necessidade de mais incentivos em pesquisas científicas, recursos humanos e melhor ambiente de trabalho para a classe. Sendo a atual revisaõ integrativa importante para estimular uma reflexão crítica, sobre futuros protocolos e políticas públicas que visem minimizar os problemas indentificados nessa pesquisa.

\section{Referências}

Alves, R. J. C., \& Ferreira, M. B. (2020). COVID-19: Reflexão da atuação do enfermeiro no combate ao desconhecido. Foco Enferm. Especial: 11 (1), $74-77$. Barbosa, D. J. et al. (2020). Fatores de estresse nos profissionais de enfermagem no combate à pandemia da COVID-19: Síntese de Evidências. Com. Ciências Saúde, 1 (31), 31-47.

Borges, E. M. das N., Queirós, C. M. L., Vieira, M. F. S. P., Teixeira, A. A. R. (2021). Percepções e vivências de enfermeiros sobre o seu desempenho na pandemia da COVID-19. Ver Rene., 22:e60790. 
Research, Society and Development, v. 10, n. 15, e186101522918, 2021

(CC BY 4.0) | ISSN 2525-3409 | DOI: http://dx.doi.org/10.33448/rsd-v10i15.22918

Botelho, L. L. R., Cunha, C. C. de A., Macedo, M (2011). O método da revisão integrativa nos estudos organizacionais. Gestão e Sociedade, 5 (11), $121-136$.

Ferreira, C. M; Almeida, D. D. C de; Mattos, M. L. A. D de; Oliveira, T. K de B. de. (2020). COVID 19: Relação do padrão epidemiológico de COVID-19 entre China e Itália. Research, Society and Development, 9 (8),1-21, e754974840.

Góes, F. G. B. et al. (2020). Challenges faced by pediatric nursing workers in the face of the COVID-19 pandemic. Rev. Latino-Am. Enfermagem, 28 :e3367.

Horta, R. L. (2021). O estresse e a saúde mental de profissionais da linha de frente da COVID-19 em hospital geral. J Bras Psiquiatr., 70 (1), $30-8$.

Humerez, D. C., Ohl, R. I. B., \& Silva, M. C. N. (2020). Saúde mental dos profissionais de enfermagem do Brasil no contexto da pandemia covid-19: ação do conselho federal de enfermagem. Cogitare Enfermagem, 25(1). doi: https://doi.org/10.5380/ce.v25i0.7411

Ludwig, F. S, et al. (2020). Pandemia da COVID-19: percepção dos profissionais de saúde sobre a assistência aludida em mídia televisiva. Rev Bras Enferm. 74 (Suppl 1), e20201258. https://doi.org/10.1590/0034-7167-2020-1258 e20201258.

Miranda, F. B. G. et al. (2020). Sofrimento psíquico entre os profissionais de enfermagem durante a pandemia da COVID-19: Scoping Rewiel. Esc. Anna Nery.25(spe):e20200363.

Moraes, E. B, et al. (2020). Safety of health professionals in COVID-19 times: a reflection. Research, Society and Development, 9 (7): 1-15,e134973832.

Moreira, A. S \& Lucca, S. R de. (2020). Apoio psicossocial e saúde mental dos profissionais de enfermagem no combate à COVID-19. Enferm. Foco., 11 (1), $155-161$.

Pereira, M. D. et al. (2020). Sofrimento emocional dos Enfermeiros no contexto hospitalar frente à pandemia de COVID-19. Research, Society and Development, $9(8), 1-21,24$.

Queiroz, A. M. et al. (2021) O ‘NOVO’ da COVID-19: impactos na saúde mental de profissionais de enfermagem? Acta Paul Enferm., 34 :eAPE02523.

Reis, L. M., Lago, P. N., Carvalho, A. H. S et al. (2020). Atuação da enfermagem no cenário da pandemia COVID-19. Revista Nursing, 23 (269): $4765-4768$.

Santos, K. M. R. dos et al. (2021). Depressão e ansiedade em profissionais de enfermagem durante a pandemia da covid-19. Esc Anna Nery, 25(spe):e20200370

Santana, N. (2020). Segurança dos profissionais de saúde no enfrentamento do novo coronavírus no Brasil. Esc Anna Nery, 24(spe):e20200241.

Souza, J. B. (2021). Círculo de cultura virtual: promovendo a saúde de enfermeiros no enfrentamento da COVID-19. Ver Gaúcha Enferm., 42(esp):e20200158.

Souza, M. T. de., Silva, M. D. da., Carvalho, R. de. (2010). Revisão integrativa: o que é e como fazer. Einsten, 8(1), 102-106.

Teixeira, C. F. de S. et al. (2020). A saúde dos profissionais de saúde no enfrentamento da pandemia de Covid- 19. Ciência \& Saúde Coletiva, 25(9):3465-3474.

Toescher, A. M. R. et al. (2020). Saúde mental de profissionais de enfermagem durante a pandemia de COVID-19: recursos de apoio. Esc Anna Nery, 24(spe):e20200276.

Ursi, E. S \& Galvão, C. M. (2006). Prevenção de lesões de pele no perioperatório: revisão integrativa da literatura. Rev Latino-am Enfermagem, 14(1):124-31. 